\title{
Percepciones docentes en torno a la evaluación del aprendizaje en el nivel educativo superior: el caso de la UABC (México) y la UCM (España)
}

Teacher perceptions around the evaluation of learning at the higher educational level: the case of the UABC (Mexico) and the UCM (Spain)

\section{Volumen 20, Número 1 \\ Enero - Abril \\ pp. 1-23}

\author{
Patricio Henríquez Ritchie \\ Brenda Boroel Cervantes \\ Vicente Arámburo Vizcarra
}

\section{Citar este documento según modelo APA}

Henríquez Ritchie, Patricio, Boroel Cervantes, Brenda y Arámburo Vizcarra, Vicente. (2020). Percepciones docentes en torno a la evaluación del aprendizaje en el nivel educativo superior: el caso de la UABC (México) y la UCM (España). Revista Actualidades Investigativas en Educación, 20(1), 1-23. Doi. 10.15517/aie.v20i1.40122 


\title{
Percepciones docentes en torno a la evaluación del aprendizaje en el nivel educativo superior: el caso de la UABC (México) y la UCM (España)
}

Teacher perceptions around the evaluation of learning at the higher educational level: the case of the UABC (Mexico) and the UCM (Spain)

\section{Patricio Henríquez Ritchie Brenda Boroel Cervantes Vicente Arámburo Vizcarra}

\begin{abstract}
Resumen. Durante el período 2017-18 se realizó una investigación cuyo objetivo general fue analizar las estrategias de enseñanza y los métodos de evaluación del aprendizaje del profesorado universitario en contextos de formación de agentes educativos. Para esto, se consideró la licenciatura en Ciencias de la Educación de la Universidad Autónoma de Baja California (UABC), México y la Facultad de Educación de la Universidad Complutense de Madrid (UCM), España. La investigación optó por un acercamiento metodológico cualitativo, a partir de lo cual se realizaron entrevistas semi-estructuradas a 19 docentes de la UCM y 5 docentes de la UABC. Con base en los objetivos del estudio, se identificaron 3 dimensiones (estrategias de enseñanza, estrategias de aprendizaje y evaluación del aprendizaje), 6 subdimensiones y 18 categorías de análisis. El presente artículo reporta los resultados relacionados con la dimensión de evaluación del aprendizaje, dentro de la cual se identificaron dos subdimensiones (funciones y estrategias/instrumentos de evaluación), siete categorías y cuatro sub-categorías de análisis. A partir de los resultados generados por esta investigación se espera aportar elementos que ayuden comprender de mejor manera las estrategias de evaluación del aprendizaje utilizadas actualmente por el profesorado universitario que forma agentes educativos y los mecanismos evaluativos funcionales al mejoramiento del aprendizaje estudiantil, especialmente de aquellos que al finalizar su carrera estarán involucrados en los procesos de enseñanza-aprendizaje de las futuras generaciones.
\end{abstract}

Palabras clave: estrategias enseñanza, estrategias de aprendizaje, estrategias de evaluación del aprendizaje

\begin{abstract}
During the period 2016-17, a research was carried out whose general objective was to analyze the teaching strategies and the methods of evaluation of the learning of the university teachers in contexts of training of educational agents. For this, the degree in Educational Sciences of the Universidad Autónoma de Baja California (UABC), Mexico and the Faculty of Education of the Universidad Complutense de Madrid (UCM), Spain were considered. The research opted for a qualitative methodological approach to the subjects involved, from which semi-structured interviews were conducted with 19 teachers from the UCM and 5 teachers from the UABC. Based on the objectives of the study and the perceptions provided by the participants, 3 dimensions were identified (teaching strategies, learning strategies and learning assessment), 6 subdimensions and 18 categories of analysis. This article reports the results related to the evaluation of learning dimension, within which two subdimensions (functions and strategies/evaluation instruments), seven categories and four sub-categories of analysis were identified. Based on the results generated by this research, it is expected to provide elements that help to better understand the learning assessment strategies currently used by university teachers that form educational agents and the functional evaluation mechanisms to improve student learning, especially those who, at the end of their careers, will be involved in the teaching-learning processes of future generations.
\end{abstract}

Key words: teaching strategies, learning strategies, evaluation of learning strategies

1Universidad Autónoma de Baja California, México. Dirección electrónica: phenriquez@uabc.edu.mx

2Universidad Autónoma de Baja California, México.Dirección electrónica: brenda@uabc.edu.mx

3Universidad Autónoma de Baja California, México.Dirección electrónica: vicente@uabc.edu.mx

Artículo recibido: 12 de junio, 2019

Enviado a corrección: 17 de setiembre, 2019

Aprobado: 14 de octubre, 2019

Los contenidos de este artículo están bajo una licencia Creative Commons 


\section{Introducción}

La implementación de estrategias de enseñanza y formas de evaluación del aprendizaje en los procesos educativos es un tema que toma ribetes particulares dentro de la educación superior. En efecto, como lo asevera Soriano Rodríguez (2012), en este nivel educativo se observa un predominio de clases expositivas en las que el profesorado transmite contenidos a sus estudiantes y privilegia la aplicación de exámenes individuales con orientaciones normativas y finalidades sumativas, aunque en algunos casos recurriendo a la retroalimentación como parte del proceso de evaluación. En este contexto, el rol estudiantil remite principalmente a aprendices que dependen de las situaciones instruccionales organizadas y protagonizadas por docentes.

Desde la perspectiva de Monereo, Castelló, Clariana, Palma y Pérez (2007), las estrategias de enseñanza impactan directamente en el proceso de aprendizaje del estudiantado: en efecto, se asevera que las estrategias de aprendizaje están directamente relacionadas con los propósitos, implícitos o explícitos, que se propone el profesorado a través del despliegue de sus estrategias de enseñanza. En otras palabras, estas últimas determinan las estrategias de aprendizaje que aplican la población estudiantil, ya que los objetivos a los que apunta la acción docente buscan promover determinadas formas de aprendizaje. Además, como lo destacan Padilla Carmona y Gil Flores (2008), las estrategias de aprendizaje también se ven directamente influidas por los métodos de evaluación utilizados por el profesorado. De esta manera, se puede aseverar que el modo de evaluar, así como las estrategias e instrumentos, condicionan las estrategias de estudio que utiliza el alumnado, por lo que la práctica evaluativa también puede considerarse una actividad de aprendizaje (Álvarez Méndez, 2001).

El presente artículo se interesa en profundizar la comprensión de las percepciones docentes en torno a los procesos de evaluación del aprendizaje, tanto en términos de las funciones que le asignan en su práctica académica como en cuanto a las estrategias e instrumentos que utilizan con sus estudiantes. Para esto, se toman como referencia dos contextos de formación de agentes educativos a nivel de educación superior: la licenciatura en Ciencias de la Educación de la Universidad Autónoma de Baja California (UABC, México) y la Facultad de Educación de la Universidad Complutense de Madrid (UCM, España). Dentro de estos contextos, se privilegió un camino metodológico cualitativo por medio de entrevistas semiestructuradas a docentes de ambas instituciones de educación superior.

El conocimiento de las estrategias de enseñanza-aprendizaje, así como de los 
métodos de evaluación que se utilizan actualmente en las licenciaturas/facultades de educación, es de suma importancia ya que permite obtener un panorama general acerca de cómo se están dirigiendo, coordinando, ejecutando y tutorando los procesos educativos a nivel universitario. Esto tiene repercusiones sociales insoslayables, ya que este estudiantado tendrá a cargo la formación de las nuevas generaciones en un futuro próximo. Por lo tanto, se debe conocer de qué manera se están aplicando las estrategias de evaluación del aprendizaje hoy en día y cuál es la percepción del profesorado universitario en la actualidad.

El objetivo del estudio fue analizar la percepción docente en torno a las estrategias de evaluación del aprendizaje que utilizan actualmente en la licenciatura en Ciencias de la Educación de la UABC y de la Facultad de Educación de la UCM.

\section{Referentes teóricos}

La evaluación del aprendizaje, independientemente del nivel educativo, se debe comprender más allá de la aplicación de exámenes y la clasificación de estudiantes a través de una escala calificativa. En este sentido, así como la realización de una tarea individual o la implementación de un trabajo de investigación grupal, se trata de una instancia más de aprendizaje en el estudiantado (Álvarez Méndez, 2001). De esta manera, se puede comprender desde su función formativa pues debe estar al servicio de quienes aprenden: el profesorado debe evaluar para conocer y para apoyar, colaborar y retroalimentar el proceso de aprendizaje de sus estudiantes, quienes continuarán aprendiendo a partir de las estrategias evaluativas aplicadas por sus profesores.

De esta manera, según la perspectiva de ciertos autores (Martínez, 2004; Monereo et al. 2007; Kamboj y Kumar, 2015) las estrategias de enseñanza que utiliza el profesorado, y por ende las estrategias de evaluación del aprendizaje, deben considerar una serie de factores asociados al perfil cognitivo de sus estudiantes: las diferencias individuales, los procesos de interacción que se desarrollan entre la planta docente y la población estudiantil, la naturaleza y complejidad de los contenidos (declarativos, procedimentales, actitudinales), entre otros factores. Además, se considera que las características personales del estudiantado (desarrollo, conocimientos previos, factores motivacionales) o su contexto intersubjetivo (conocimiento compartido), también son elementos que deben ser considerados en la acción docente a la hora de elegir las estrategias de enseñanza y de evaluación del aprendizaje.

No obstante lo anterior, en la actualidad existe una persistencia insoslayable de la 
función sumativa en los procesos de evaluación del aprendizaje. En efecto, desde que Scriven propuso hacia mediados de la década de 1960 las dos funciones, sumativa y formativa, de la evaluación, se ha desarrollado un intenso debate acerca de cómo enfocar los procesos evaluativos del aprendizaje del estudiantado (Chiva, Perales y Pérez Carbonell, 2009; Ramos, Perales y Pérez, Carbonell, 2009; Carbajosa, 2011). Mientras por un lado se aboga por la arista sumativa de dichos procesos, por otro lado se defiende la perspectiva formativa de la evaluación del aprendizaje.

A grandes rasgos, la función sumativa de la evaluación apunta a informar acerca de los resultados finales del proceso educativo, determinando la calidad, productividad, costos y efectos de la realidad evaluada. Por lo tanto, se considera como una evaluación final y se relaciona con la rendición de cuentas y toma de decisiones: aprobación/reprobación, acreditación/no acreditación, aceptación/rechazo, entre otros. Por su parte, la función formativa de la evaluación se caracteriza por valorar más los procesos que los resultados finales, apuntando al mejoramiento y retroalimentación constante de la realidad evaluada. Para esto, se proponen diversos momentos o instancias de evaluación (diagnóstica, implementación, resultados) y una toma de decisiones continua, no sólo al finalizar un proceso (Ramos, Perales y Pérez, Carbonell, 2009; Martínez Rizo, 2012).

En base a esta dualidad sumativa/formativa de funciones de la evaluación, o, en palabras de Álvarez Méndez (2001) entre la evaluación desde "la racionalidad práctica" y "la racionalidad técnica", la tendencia principal hacia las últimas décadas es destacar la función formativa en desmedro de la sumativa. Sin embargo, la realidad dista mucho del discurso. En efecto, algunos estudios (Moreno Olivos, 2007; Instituto Nacional para la Evaluación de la Educación [INEE], 2011; García Sanz, 2014; Zitzelsberguer, Coffey, Graham, Papaconstantinou y Anyiman, 2017) han destacado que las prácticas evaluativas en la actualidad están guiadas por su dimensión sumativa, más allá de la valoración formativa observada a nivel oficial. En este sentido, gran parte de la población docente se mantiene bajo la perspectiva de comprender la evaluación como sinónimo de medición (calificación), mientras que el estudiantado tiende a centrar su interés en el estudio exclusivo de los contenidos que se incluyen en los exámenes de evaluación.

Por su parte, respecto a los instrumentos de evaluación, se debe destacar que no existen instrumentos inherentemente sumativos o formativos: lo que efectivamente existe es la función sumativa o formativa de la evaluación de acuerdo a cómo utiliza la información la 
población docente. Un examen individual con preguntas de opción múltiple, por ejemplo, no es sumativo por sí solo. Así como un ensayo o un trabajo de investigación grupal tampoco es inherentemente formativo. La connotación de sumativo o formativo la otorga el uso que se hace de la información recogida a través de dichos instrumentos, independientemente de cual sea: si califica a través de una escala numérica y establece medias de calificación para agrupar al estudiantado aprobado y reprobado o si aplica un sólo instrumento al final del período escolar para determinar quién aprueba y quién no, estaríamos hablando de la función sumativa de la evaluación. Por el contrario, si aplica varios instrumentos (exámenes diagnósticos, exámenes individuales, ensayos grupales, trabajos de investigación, etc.) dentro del período escolar y posteriormente a cada aplicación se detiene a analizar los resultados grupales para retroalimentar aquellos aspectos en que el estudiantado demuestra baja comprensión, estaríamos frente a la función formativa de la evaluación del aprendizaje.

El presente artículo expone los resultados parciales de una investigación cuyo objetivo general fue analizar las estrategias de enseñanza y métodos de evaluación del aprendizaje desde la percepción docente, profundizando en 3 dimensiones principales: i) estrategias de enseñanza, ii) estrategias de aprendizaje y iii) evaluación del aprendizaje. Acá se muestran los resultados de esta última dimensión, dividida en dos subdimensiones de análisis: i) funciones de la evaluación del aprendizaje y ii) estrategias e instrumentos de evaluación del aprendizaje.

\section{Método}

\subsection{Enfoque}

El enfoque de la investigación, entendido como la estrategia de acción utilizada durante el estudio para recolectar información, fue cualitativo con un nivel de alcance exploratorio. Dentro de este enfoque, se priorizó un diseño fenomenológico, dado que las preguntas que guiaron el estudio indagaron sobre la esencia de las experiencias del profesorado universitario en torno al uso de estrategias de evaluación del aprendizaje, a la vez que se pretendió describir sus experiencias personales al respecto (Hernández Sampieri, Fernández Collado y Baptista Lucio, 2014)

\subsection{Unidades de análisis}

La investigación se llevó a cabo en dos instituciones de educación superior relacionadas con la oferta de programas de formación de agentes educativos: i) la UABC, 
Facultad de Ciencias Administrativas y Sociales (FACYS), licenciatura en Ciencias de la Educación, y ii) la UCM, Facultad de Educación. La licenciatura en Ciencias de la Educación de la UABC ofrece un programa enfocado en la formación de profesionales involucrados en la investigación/gestión/administración de la educación. La Facultad de Educación de la UCM consta de 9 departamentos enfocados en la formación del profesorado: i) didáctica de la lengua y literatura, ii) didáctica de las ciencias experimentales, iii) didáctica de las ciencias sociales, iv) didáctica de las matemáticas, v) didáctica y organización escolar, vi) expresión musical y corporal, vii) métodos de investigación y diagnóstico en educación, viii) psicología evolutiva y de la educación y ix) teoría e historia de la educación. El contraste de la magnitud y de la oferta de programas educativos, así como su convergencia en torno al ámbito de las ciencias educativas, son los elementos principales que justifican la elección de ambos escenarios en la investigación. A su vez, el proyecto se realizó durante el período 2017-18.

Según registros oficiales de la $\mathrm{UABC}$, durante el período de la investigación la licenciatura en Ciencias de la Educación de la UABC constó una planta docente de tiempo completo de 6 profesionales, quienes cumplen tanto labores de docencia como de investigación y coordinación de programas académicos o de extensión. Además, trabajaban 19 profesionales que cumplen sólo funciones de docencia, sin involucrarse en investigación ni coordinación dentro de la universidad. A partir de la accesibilidad de la planta docente, el presente estudio seleccionó una muestra intencional no probabilística de 4 profesores de tiempo completo y 1 de asignatura de este programa educativo.

Por su parte, los departamentos de la Facultad de Educación de la UCM están formados por un promedio de 25 profesionales de tiempo completo, de lo que deriva una población de más de 200 docentes-investigadores. De estos, se seleccionó una muestra intencional no probabilística de 19 docentes, considerando todos los departamentos de esta Facultad. En síntesis, la presente investigación incluyó una muestra intencional no probabilística de 24 docentes, entre ambos escenarios seleccionados (tabla 1). Respecto a la gran diferencia entre la muestra de docentes de la UCM y la UABC, cabe mencionar que se debe principalmente a las diferencias entre la magnitud de las poblaciones de cada contexto. Además, debido a la naturaleza exploratoria y cualitativa del estudio, no se pretenden realizar comparaciones entre ambos escenarios, las cuales serían inválidas debido a las diferencias antes mencionadas. A su vez, cabe destacar que se obtuvo el consentimiento de cada uno de los participantes para exponer sus percepciones guardando la confidencialidad exigida por la ética de la práctica investigativa. 
Tabla 1. Departamentos y número de participantes de la Facultad de Educación (UCM) y de la licenciatura en Ciencias de la Educación (UABC), 2017-18

\begin{tabular}{|l|c|}
\hline \multicolumn{1}{|c|}{ DEPARTAMENTOS (UCM) } & PARTICIPANTES \\
\hline DIDÁCTICA DE LAS CIENCIAS SOCIALES (CCSS) & 3 \\
\hline DIDÁCTICA DE LAS CIENCIAS EXPERIMENTALES (CCEE) & 2 \\
\hline DIDÁCTICA DE LA LENGUA Y LITERATURA (LENGUA Y LIT) & 3 \\
\hline DIDÁCTICA DE LAS MATEMÁTICAS (MAT) & 2 \\
\hline DIDÁCTICA Y ORGANIZACIÓN ESCOLAR (DIDAC) & $\mathbf{2}$ \\
\hline TEORÍA E HISTORIA DE LA EDUCACIÓN (TEO E HISTO) & $\mathbf{3}$ \\
\hline EXPRESIÓN MUSICAL Y CORPORAL (MUS Y CORP) & 1 \\
\hline MÉTODOS DE INVESTIGACIÓN Y DIAGNÓSTICO EN EDUCACIÓN \\
(MIDE) & 19 \\
\hline PSICOLOGIA EVOLUTIVA Y DE LA EDUCACIÓN (PSICO Y EVO) & 5 \\
\hline SUBTOTAL & 24 \\
\hline LIC. EN CIENCIAS DE LA EDUCACIÓN (UABC) & \\
\hline TOTAL & \\
\hline Fuente: elaboración propia (2018) & \\
\hline
\end{tabular}

\subsection{Técnicas de recolección}

Se utilizó la entrevista en profundidad como técnica de recolección de información. Esta técnica es considerada como una herramienta de gran utilidad en los estudios sociales para acceder a algunos ámbitos subjetivos de las personas (percepciones, valoraciones en torno a situaciones, intereses, proyecciones, creencias), permitiendo la interpretación de la realidad social y transformándolas en interlocutoras sociales a través del diálogo y la interacción (Robles, 2011; Vargas Jiménez, 2012).

De esta manera, se elaboró una pauta de entrevista semiestructurada con el objetivo de guiar y enfocar el diálogo en función de los objetivos del estudio. La pauta establece 3 dimensiones y 6 subdimensiones en torno a la temática de la investigación. Para efectos del presente documento, se muestra la dimensión a la que remite la temática asociada y las subdimensiones correspondientes (tabla 2):

Tabla 2. Pauta de entrevista semiestructurada para docentes

\begin{tabular}{|c|c|c|}
\hline Dimensión & Subdimensiones & Preguntas \\
\hline \multirow{3}{*}{$\begin{array}{l}\text { Evaluación } \\
\qquad \text { del } \\
\text { aprendizaje }\end{array}$} & Funciones & $\begin{array}{l}\text { - ¿Cuáles son las funciones que tiene (debe tener) la evaluación dentro del } \\
\text { proceso de enseñanza-aprendizaje? }\end{array}$ \\
\hline & Estrategias & $\begin{array}{l}\text { - ¿Cuáles son las estrategias de evaluación que utiliza en el marco de su } \\
\text { práctica de enseñanza? ¿Con qué finalidad? } \\
\text { - ¿De qué manera afecta tamaño del grupo (cantidad) de estudiantes en las } \\
\text { estrategias de evaluación que utiliza? }\end{array}$ \\
\hline & Instrumentos & - ¿Qué instrumentos de evaluación utiliza en su práctica docente? \\
\hline
\end{tabular}




\subsection{Procesamiento de análisis}

Las etapas de desarrollo de la investigación se organizaron en función de la estructuración teórica y metodológica del estudio. De esta manera, se siguieron las siguientes fases:

- Desarrollo de antecedentes y marco teórico (septiembre-octubre 2017). Elaboración del marco teórico del estudio, estructuración de las bases conceptuales y definición de conceptos claves.

- Aplicación de entrevistas en profundidad (noviembre 2017-febrero 2018). Una vez solicitados los permisos correspondientes, se procedió a aplicar el instrumento de recogida de información. En una primera instancia, la aplicación se realizó en la licenciatura en Ciencias de la Educación de la FCAYS de la UABC, por ser el escenario más cercano al contexto espacial del estudio. Posteriormente, en el período enero-febrero de 2018 se llevaron a cabo las entrevistas a la planta docente de Facultad de Educación de la UCM. A nivel general, las entrevistas implicaron una interacción dialógica cuyo tiempo de duración fue de 40 minutos en promedio por cada docente.

- Análisis de resultados (marzo-agosto 2018). Durante esta etapa se realizó el análisis cualitativo de la información recolectada.

La información recolectada a través de las entrevistas se analizó a través del análisis de contenido. Esta técnica permite interpretar los datos recogidos a través del levantamiento de códigos y categorías identificadas en los discursos de las personas. De esta manera, como lo asevera Piñuel Raigada (2002), remite a un conjunto de procedimientos interpretativos de productos comunicativos tales como mensajes, textos o discursos, originados en procesos dialógicos previamente registrados (en nuestro caso, entrevistas en profundidad). Los pasos seguidos en el análisis cualitativo de la información fueron:

- Captura de la información mediante grabaciones de audio de las entrevistas.

- Realización de transcripciones.

- Análisis de las opiniones, percepciones y discursos, a través de la técnica de análisis de contenido.

- Estructuración de la información a través de la identificación de categorías (clasificación conceptual de unidades temáticas cubiertas bajo un mismo tópico) y códigos (conjunto de unidades de texto que corresponden dentro de 
una categoría de análisis). Las categorías fueron identificadas a través del análisis de contenido con base en las entrevistas realizadas, levantándose las siguientes: función sumativa, función formativa, equilibrio entre funciones, estrategias individuales, grupales, complementariedad de estrategias y factores en torno al uso de estrategias e instrumentos de evaluación del aprendizaje.

\section{Resultados}

El análisis información recogida en torno a las estrategias de evaluación del aprendizaje desde la percepción docente, derivó en la identificación dos subdimensiones: i) funciones de la evaluación del aprendizaje y ii) estrategias e instrumentos de la evaluación del aprendizaje.

\subsection{Subdimensión: funciones}

A través de esta subdimensión se intentó explorar los objetivos y las funciones que el profesorado le asigna a la evaluación del aprendizaje, así como también su manera de comprenderla en el marco de su actividad de enseñanza y las estrategias utilizadas al respecto. A partir de sus percepciones, se identificaron 3 categorías de análisis: i) función sumativa, ii) función formativa y iii) equilibrio entre funciones sumativas y formativas.

1) Función sumativa: desde la perspectiva docente, la evaluación del aprendizaje debe tener una función insoslayablemente sumativa en términos de control, toma de decisiones y certificación social. En efecto, tal como lo propuso Scriven hacia mediados de la década de 1960, una de las funciones que se le asigna a las prácticas evaluativas en contextos educativos, principalmente en términos de aprendizaje, remite a controlar los procesos de adquisición de conocimientos, tomar decisiones en términos de aprobación/reprobación y certificar socialmente al alumnado una vez que termina sus estudios. De esta manera, se identificaron 2 subcategorías de análisis: i) toma de decisiones en base a exámenes finales y ii) certificación social.

- Toma de decisiones con base a exámenes finales: desde la percepción de algunos docentes entrevistados, se comprende la evaluación del aprendizaje desde su función sumativa, principalmente asociada a la aplicación de exámenes objetivos al final del período académico para determinar la aprobación/reprobación según sus 
resultados. En efecto, se asevera que las prácticas evaluativas apuntan a determinar el aprendizaje de todo el contenido teórico de la materia, estableciendo la aprobación/reprobación del estudiantado con base en una calificación final:

“... Hacemos una prueba objetiva, sólo una al final del curso, donde entra toda la materia en contenidos digamos teóricos, un examen de opción múltiple, siento que bastante bien elaborada porque pues sé elaborar, trabajo en esto en esto a nivel de investigación... Lo que hago claramente es sumativo, quiero decir, yo me espero al final y se decide si sí o si no... Yo hago la evaluación solo para dar una calificación, esa es la realidad, si no, no lo haría..." (docente A, MIDE, UCM).

“... En realidad casi todos mis dispositivos de evaluación tienen que ser sumativos... No sé si me identificaría más, en el plano técnico-conceptual, pero acá estoy hablando no de lo que creo que debería hacer, sino de lo que hago y bueno, pues si, respondiendo con honestidad. Obviamente que yo participo con todo el universo docente en lo importante que es la evaluación formativa, lo importante que es el feedback al estudiante, lo importante del acompañamiento durante la construcción de los aprendizajes... En realidad, te estoy diciendo lo que hago...” (docente C, MIDE, UCM).

- Certificación social: asociado a lo anterior, se comprende la evaluación a partir de su función social, la cual remite a la certificación social profesional del estudiantado (González Pérez, 2001; Rizo Moreno, 2004). En este sentido, se destaca que, ante la inexistencia de organismos encargados de certificar profesionalmente a la población egresada del nivel educativo superior, la evaluación del aprendizaje debe cumplir con el rol de asegurar a la sociedad su calidad profesional en cualquier campo del conocimiento, particularmente en el ámbito de la educación y de la futura población docente, pues será la que tendrá a cargo la educación de las nuevas generaciones:

“... Las instituciones universitarias tienen una característica importante, es que tienen un valor de no sólo formación general humanística, sino también es una formación profesional. Además lo que ocurre en España, por ejemplo, no hay como en otros países una prueba para conseguir una certificación profesional que te habilite para la profesión, ya en España cuando terminas tus estudios estás habilitado profesionalmente para ejercer. Entonces, claro, debe de haber necesariamente una evaluación sumativa que garantice a la sociedad a quien egresa la certificación..." (docente B, MIDE, UCM); “... Evidentemente al final hay que evaluar porque tú les 
acreditas para una función, entonces le aseguras a la sociedad que esta persona ha pasado por tal, entonces hay una parte ahí que es una responsabilidad que tengo que tomar..." (docente C, TEO E HIST, UCM).

2) Función formativa: paralelamente, desde la percepción docente también se destaca la función formativa de la evaluación del aprendizaje, enfocándose en la implementación de evaluaciones diagnósticas, en la verificación y mejoramiento del aprendizaje, así como también en la retroalimentación, monitoreo y apoyo constante al estudiantado en su proceso de construcción de conocimientos:

“... Sí, la orientación es formativa, no a la memorización... No podemos pedir que se memorice lo que ya está en las máquinas, lo que es importante es que tengan capacidad de construcción de temas, de establecer relaciones, de comparar y eso me parece que es madurez intelectual y madurez profesional también...” (docente B, CCSS, UCM).

“... Yo pensaría que me inclino más hacia lo formativo, tratar de que lo que se está viendo en clase se aprenda y de alguna manera irlo evaluando, a través, por ejemplo de preguntas, de ejemplos, a través de que ellos contesten... Al principio les hago una evaluación un poco diagnóstica para ver que tanto saben, entonces, se supone que en las materias que uno imparte ya hay una materia que les antecedió, donde ya se revisaron algunos conceptos, la mayoría de las veces hay que regresarse y volverlos a ver..." (docente A, UABC).

En relación con lo anterior, para algunos docentes el manejo de la información recolectada a través de los instrumentos de evaluación del aprendizaje que aplican a sus estudiantes se enfoca hacia fines totalmente formativos, principalmente en función de la retroalimentación y el mejoramiento/monitoreo/apoyo al aprendizaje. Al respecto, se destaca que dentro de los instrumentos utilizados, se promueve la aplicación de exámenes individuales por normativa institucional. Sin embargo, la función que asignan a los resultados de la evaluación es eminentemente formativa. De esta manera, según esta perspectiva la orientación sumativa o formativa de la evaluación del aprendizaje estaría determinada por el manejo que hace el profesorado de la información recolectada, más allá del instrumento de evaluación utilizado:

“... Yo me identificaría más, o me gustaría identificarme más, con el formativo y el problema que estoy viendo es cuanto más gente tengas en clase, más difícil es estar viendo el seguimiento y entonces como profesor lo paso mal, me da mucha rabia que se 
siga todavía poniendo tanto énfasis en el examen final, odio los exámenes finales, no me gustan porque creo que muchas veces la gente tiene un día malo y entonces eso de tener que estar por narices teniendo en cuenta tal porcentaje de tal nota para una cosa concreta, me parece un tanto contradictorio con todo el discurso educativo que se está dando ahora, nos contradecimos a nosotros mismos ..." (docente C, LENGUA Y LIT, UCM).

3) Equilibrio entre funciones sumativas y formativas: a nivel general, a partir de la percepción docente se observa un posicionamiento equidistante entre las funciones sumativas y formativas de la evaluación del aprendizaje. En este sentido, se destaca la consideración de los elementos formativos para complementar la toma de decisiones en base a una calificación final aprobatoria o reprobatoria, adaptando las estrategias evaluativas para no afectar negativamente al estudiantado que ve mermada su calificación basada en exámenes finales de opción múltiple, asociados a la función sumativa de la evaluación. De esta manera, se asevera que se deben mezclar funciones formativas y sumativas en los métodos de evaluación utilizados en la práctica docente: además de llegar a una calificación individual final (aprobación o reprobación), consideran que se debe ir evaluando durante todo el proceso educativo a través de instrumentos heterogéneos: trabajos grupales, ensayos, exposiciones grupales, exámenes individuales, entre otros:

“... Mi idea es, algunas veces el artefacto con el que mides puede estar influyendo en la medida, por esa razón siento que aquellas personas que no les va bien el modo de estudiar para un examen de prueba objetiva no les va bien, porque sienten mucha angustia, por lo que sea, se arriesgan demasiado, no saben equilibrar, etc., que tengan la oportunidad de complementar esa nota con otro modo de examinarse, porque si tienen los conocimientos y los expresan de otra manera..." (docente A, MIDE, UCM).

“... Tienes que hacer dos exámenes parciales, entonces igual esas evaluaciones no necesariamente son exámenes de opción múltiple. Por ejemplo, les puedo dejar una línea de tiempo, un ensayo, un examen a libro abierto. Entonces dependiendo de los contenidos, si son muchos contenidos prefiero dejarles un examen a libro abierto donde puedan identificar lo que más ocupo que refuercen, en ocasiones si aplico la memorización en determinados datos, ocupo que sepan que existe tal artículo de educación y que sepan el fundamento teórico y si quiero que conozcan ese artículo y si 
quiero que lo apropien y más por su disciplina. Entonces si aplico la formativa junto con la sumativa, ambas..." (docente D, UABC)

Cabe destacar que a partir de las percepciones docentes en torno a las funciones que le asignan a la evaluación del aprendizaje, se observa un grado de confusión conceptual al momento de describirlas. En efecto, se hace mención a "tipos de evaluación formativa o sumativa" a partir de los instrumentos que utilizan, no de la función que le asignan al acto de evaluar ni del uso de la información recolectada. Por lo tanto, se deja entrever una concepción imprecisa de las funciones de la evaluación, las cuales se definen, por antonomasia, a partir del uso que se hace de la información recolectada, independientemente del instrumento utilizado.

\subsection{Subdimensión: estrategias e instrumentos}

Otro punto se centró en las estrategias e instrumentos de evaluación que el profesorado aplica en su práctica docente. Dentro de esta subdimensión, se identificaron cuatro categorías de análisis: i) estrategias individuales de evaluación, ii) estrategias grupales de evaluación, iii) complementariedad de estrategias e instrumentos y iv) factores que inciden en el uso de estrategias de evaluación:

1) Estrategias individuales: dentro de esta estrategia, se destaca la aplicación de ciertos medios o herramientas de evaluación tales como exámenes objetivos individuales de opción múltiple y se vislumbra una percepción positiva acerca de estos instrumentos de evaluación del aprendizaje. Al respecto, el profesorado percibe que la fiabilidad y validez de estos instrumentos les permiten asegurar la ecuanimidad al momento de evaluar, de manera que nadie se vea afectado por sesgos de medida en la calificación obtenida. Por su parte, paralelamente se destaca el uso de otros instrumentos de evaluación, tanto cuantitativos como cualitativos, relacionados con estrategias individuales, tales como exámenes tipo ensayo, exámenes orales, portafolio de evidencias y reportes de lectura, entre otros:

“... Utilizo básicamente en mi esquema de evaluación dos tipos de dispositivo de evaluación: hago una prueba escrita, un examen tipo test, de opción múltiple habitualmente con tres tipos de respuesta, de la cual sólo una es la correcta. Y yo se supone que soy experta para construirlas, así que me fio mucho de mis pruebas, entonces habitualmente siempre en mis asignaturas, los estudiantes tienen que pasar 
una prueba individual de estas características... Te decía tengo mucha confianza en mis exámenes porque creo que son pruebas bien construidas, tengo un buen banco de ítems, conozco la fiabilidad, tengo calibrado el índice de dificultad de los ítems, construyo una prueba con distintos niveles de dificultad...” (docente C, MIDE, UCM).

“... Tienen la obligación de hacer por cada capítulo un reporte de lectura y ese reporte de lectura es libre, es decir, para ellos es mejor, si ya identificaron que organizador gráfico es mejor, que lo hagan, si es síntesis que lo hagan, a veces hacen reportes tipo casos, que hacen preguntas o copian los casos de libros de texto que revisamos eso es en metodología..." (docente B, UABC).

Dentro de la categoría de estrategias individuales de evaluación del aprendizaje, hay que destacar una forma de evaluar de manera personalizada pero omitiendo el uso de instrumentos: la asistencia a clases como criterio de evaluación. En efecto, desde la percepción docente se considera la asistencia a clases como un criterio subliminal de evaluación, de manera que la inasistencia podría afectar negativamente la aprobación de la materia, respecto a la asistencia regular a clases. En este sentido, si bien no se considera un criterio directo de aprobación/reprobación (aunque no asistan a clases, el alumnado igualmente tiene derecho a un examen sumativo al final del semestre para decidir su aprobación o reprobación), se comprende como un elemento que puede ayudar a alcanzar los puntos necesarios en la calificación final para aprobar la materia: “... Yo hago lo siguiente: la asistencia es obligatoria, yo no la controlo todos los días, pero los jueves o los miércoles, que tengo prácticas, ese día si controlo la asistencia, yo digo que lo que hacen es sumar, cada alumno tiene una hoja de cada día, ellos tienen que marcar en las que estuvieron...” (docente B, MIDE, UCM).

“... Si es una persona que nunca ha venido y no lo conozco, yo creo que el que viene a clase tiene un plus, porque preguntas, escuchas a otros, te hace pensar, pero no le doy demasiada trascendencia. Hasta me parece en cierto sentido poco relevante, porque si la persona no viene nunca todavía tiene derecho a examen, es una contradicción. Bueno, si estoy poquito dudosa entre un 8 y 9, si es una persona que ha venido, que ha participado, que ha mostrado interés, si tiendo a subirla, que valoro a la persona que ha trabajado a lo largo del curso..." (docente B, LENGUA Y LIT, UCM).

2) Estrategias grupales: por su parte, desde otra arista de la percepción docente también aparece la implementación de estrategias grupales de evaluación como una categoría 
de análisis importante dentro de esta subdimensión. En efecto, se mencionan los trabajos grupales de investigación como la herramienta mayormente utilizada dentro de esta estrategia evaluativa: según esta percepción, la elaboración de estos trabajos permite superar las dificultades a las que se enfrentan tanto el profesorado como el alumnado cuando se utilizan exclusivamente exámenes individuales de opción múltiple. En este sentido, pese a los estándares psicométricos de confiabilidad y validez, se reconoce que la triangulación en el uso de instrumentos de evaluación del aprendizaje es el camino idóneo para lograr una evaluación justa e imparcial:

“... Los trabajos de investigación y ensayos, todo es equipo, los equipos, dos tipos de equipo, los de situación y los de campo: los de situación son los que haces en la clase como para alguna actividad y de campo son de todo el semestre. Los de situación yo los conformo, para cada actividad, pero para el equipo final yo no me meto...” (docente B, $\mathrm{UABC})$.

Cabe destacar que dentro de las estrategias grupales también aparece la autoevaluación grupal como estrategia evaluativa: se considera que esta herramienta permite extender el proceso de aprendizaje grupal hacia las instancias evaluativas. Al respecto, se comprende la evaluación como una actividad más de aprendizaje, pues se considera que el estudiantado que aprende a través de dinámicas grupales también estará capacitado para evaluar colectivamente a sus pares, a través de la elaboración de informes de trabajos grupales de autoevaluación:

“... La evaluación es individual, personal y grupal, el que aprende en grupo tiene que evaluar en grupo. Si yo creo que aprenden en grupo, aprenden a evaluar en grupo, entonces tienen que elaborar un informe (en este año y el año pasado, mínimo ocho mil caracteres, de entre ocho mil y diez mil caracteres) donde tienen que aplicar unos criterios que previamente han elegido y han escrito y yo les he leído y he dado el visto bueno, todos lo aplican y tienen que elaborar un informe de evaluación..." (docente B, DIDAC, UCM).

3) Complementariedad de estrategias e instrumentos: desde otra perspectiva, se percibe que un camino metodológico idóneo para evaluar al estudiantado es la complementariedad de estrategias e instrumentos, tanto grupales como individuales, de manera que la calificación final se vea permeada por ambas formas de evaluar el 
aprendizaje. Esta triangulación en el uso de estrategias e instrumentos de evaluación del aprendizaje implica la complementación de formas de evaluación individuales y grupales, así como también de instrumentos de recogida de información: trabajos, exposiciones, preguntas orales, portafolios, etc.:

“... Hay que tener en cuenta también el factor individual, porque si no a veces, creemos que nos faltan elementos, variables, a la hora de evaluar individualmente, porque la evaluación no es en conjunto, la evaluación es individual, de ahí que yo siempre he tenido un método: las estrategias de trabajo mixtas... Sistema mixto..." (docente A, CCSS, UCM).

“... En el encuadre les presento los criterios de evaluación: son exámenes, ensayos, tareas y trabajos, y trabajo final, tareas y trabajos durante el semestre y el trabajo final, pero en esas tareas y trabajos pueden ser diferentes diversidad de instrumentos, como tareas y recursos, desde un ensayo, hasta un cuadro comparativo, una línea de tiempo dependiendo, ya la conjunción de esos me da un porcentaje de tareas..." (docente B, $\mathrm{UABC})$.

4) Factores en torno al uso de estrategias e instrumentos de evaluación: por último, una cuarta categoría de análisis remite a los factores identificados en torno al uso de estrategias e instrumentos de evaluación del aprendizaje. En efecto, a partir de la percepción de algunos docentes se identifican dos conjuntos de factores: i) factor pedagógico (características cognitivas) y ii) factor extra-pedagógico (cantidad en el aula):

a) Factor pedagógico: desde algunas aristas de la percepción docente se asevera que las estrategias e instrumentos de evaluación del aprendizaje las adecúan a las características cognitivas del estudiantado. Estas características están asociadas al semestre cursado. De esta manera, en los semestres más avanzados, donde se demuestra un mayor desarrollo cognitivo y mejor manejo teórico-práctico de los contenidos, son más propicia la aplicación de evaluaciones de desarrollo de ideas (tipo ensayo) y trabajos de investigación. Por su parte, en los semestres iniciales, donde se observa una madurez cognitiva incipiente, se percibibe como más afín al uso de estrategias individuales de evaluación a través de instrumentos como exámenes de respuesta cerrada u opción múltiple:

“...En los cursos superiores me hacen un ensayo individual, entonces tienen una rúbrica para ese ensayo, la rúbrica no se la doy al principio, la doy cuando ya han 
manchado papel, han decidido el tema, han pensado bien las ideas... Eso no lo hago en los primeros cursos, primero porque me parece que todavía necesitan como elementos para poder tener juicio... Con los de primero es más importante, porque entre comillas a pesar del contenido, les tengo que domar, acaban de terminar el bachillerato, entonces hay un proceso en el que ellos te retan, porque ya son libres y adultos y su aprendizaje depende de ellos, entonces hay un proceso de concienciarles de que están en la universidad, cosa que cuando están en tercero o cuarto no es necesario..." (docente C, TEO E HIST, UCM).

“... Si yo me centrara en la materia de 4to te diría que ellos tienen más peso que yo, quiero decir en su aprendizaje, creo que ellos, si tuviera que dar porcentaje a lo mejor ellos tienen un $70 \%$ y yo en el 30 , participativa más bien de ellos, el que quiera aprender, yo estoy como guía, y obviamente si no estuviéramos sería mucho más complicado para ellos, pero yo diría que una parte importante de su aprendizaje ha dependido únicamente de su actividad..." (docente A, MIDE, UCM).

b) Factor extra-pedagógico: por su parte, según la percepción docente el tamaño de los grupos sería un factor trascendental para determinar las estrategias e instrumentos de evaluación del aprendizaje. De esta manera, una menor cantidad de estudiantes permitiría recurrir a exámenes de desarrollo tipo ensayo, poniendo más atención en la expresión de ideas y capacidad de análisis. Por su parte, una mayor cantidad de estudiantes por grupo obligaría a recurrir a exámenes de opción múltiple, para examinar principalmente la retención y comprensión de contenidos, además de ser más fáciles y rápidos de revisar. Además, se percibe que el sistema de créditos por materia adoptado por la institución universitaria también es un factor que determina las estrategias e instrumentos, dada la corta duración de cada materia y la superficialidad con que deben revisarse grandes cantidades de contenidos educativos:

“... El tamaño influye en la manera de evaluación, si fueran los grupos más chicos, seguramente sería más formativa, iría trabajando por temáticas, y evaluando cada temática, aunque haría una evaluación final porque estoy convencida, independientemente que fueran dos o diez porque creo en la 
evaluación final. Y yo creo que tendría más datos sobre cada alumno, porque yo realmente no conozco a mis alumnos..." (docente A, MIDE, UCM).

“... Lo que pasa es que los instrumentos de evaluación serían otros. En principio, no haría examen final escrito, trabajaríamos más en plan de taller, que ellos pudieran elaborar más los temas conmigo, darle más implicación a ellos, a la búsqueda de información en cada tema de cada unidad, hay varios subtemas, entonces no da tiempo a que ellos lo expliquen o lo expongan... No me haría falta hacer un examen escrito ni final porque conocería a los alumnos, porque hablaríamos permanentemente..." (docente A, DIDAC, UCM).

En la figura 1 se observa un esquema descriptivo de la dimensión evaluación del aprendizaje. Asociadas a esta dimensión, se identificaron 2 subdimensiones (funciones y estrategias/instrumentos), dentro de las cuales se levantaron 7 categorías de análisis (función sumativa, formativa, equilibrio entre funciones sumativas y formativas; estrategias individuales, grupales, complementariedad de estrategias e instrumentos, factores en torno al uso de estrategias e instrumentos) y 4 subcategorías de análisis (toma de decisiones en base a exámenes finales, certificación social; factores pedagógicos y extra-pedagógicos en torno al uso de estrategias e instrumentos de evaluación del aprendizaje): 
Figura 1. Subdimensiones y categorías de análisis en torno a la dimensión evaluación del aprendizaje

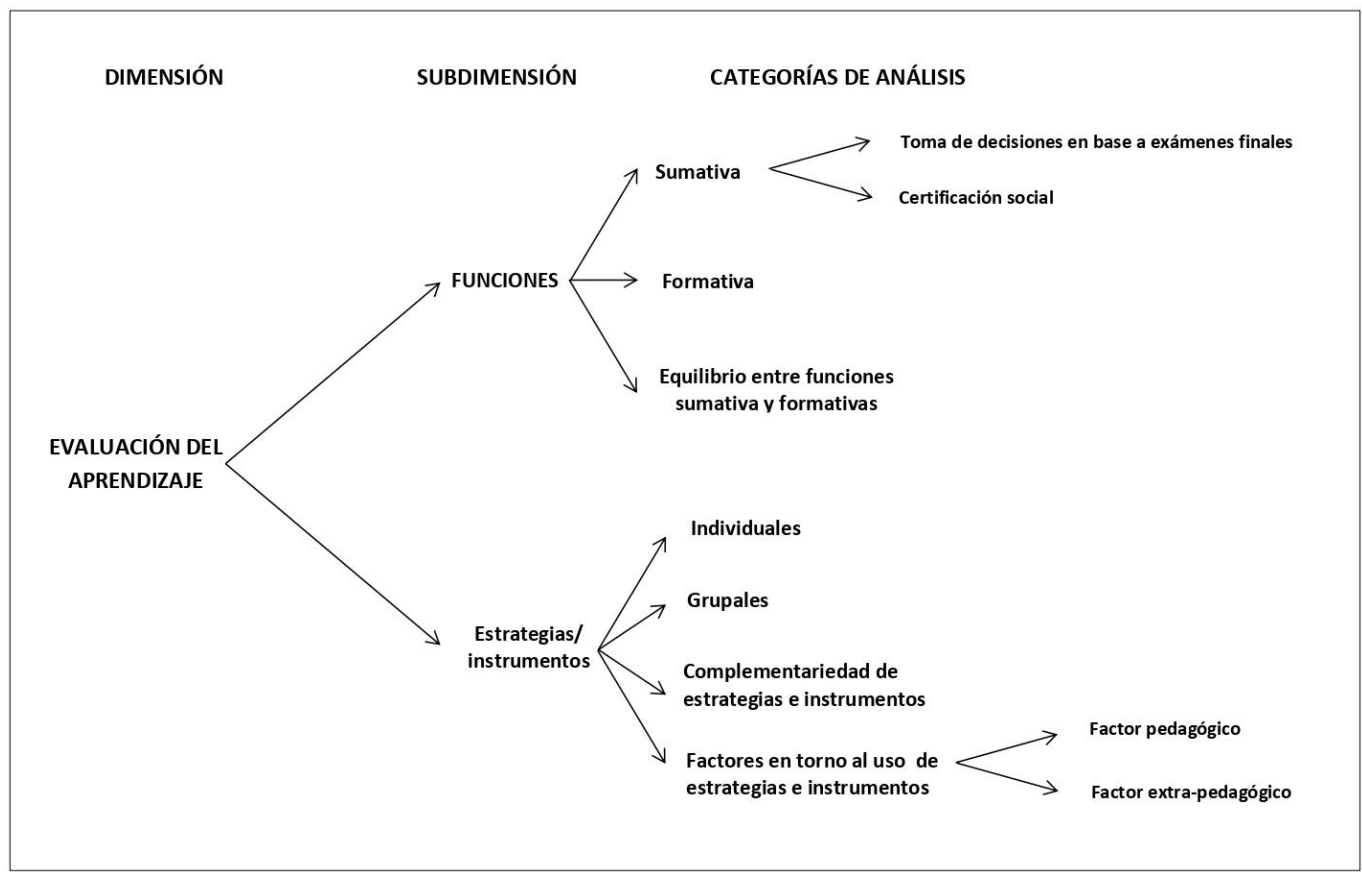

Fuente: elaboración propia (2018)

\section{Conclusiones}

La evaluación del aprendizaje, tal como lo propone Álvarez Méndez (2001), debe comprenderse como una actividad planificada por el profesorado dentro del conjunto de estrategias diseñadas y desplegadas para favorecer el aprendizaje de sus estudiantes. No se trata de una instancia separada del proceso de enseñanza-aprendizaje, sino más bien una actividad que apoya, fortalece y propicia el aprendizaje, en la medida en que se utiliza como un medio de observación, análisis y retroalimentación, alejándose de su comprensión punitiva, excluyente y exclusivamente calificadora.

Respecto a su definición conceptual y a las funciones que se le asigna en la práctica docente, se retoman los aportes de Ramos, Perales y Pérez, Carbonell (2009), que definen la evaluación como aquel proceso sistemático de recolección de evidencias o información acerca de una realidad educativa (en este caso, el aprendizaje), con el propósito de emitir juicos de valor que llevarán a dos caminos distintos pero complementarios: i) la toma de decisiones finales (función sumativa) y ii) la retroalimentación y el mejoramiento procesual de la realidad evaluada (función formativa). En el presente documento se enfatiza que la 
evaluación educativa en general y del aprendizaje en particular, debe considerar las dos funciones propuestas por Scriven hacia la década de 1960, de manera que se implementen prácticas evaluativas con funcionalidad mixta.

A nivel general y a partir del análisis de las percepciones del profesorado entrevistado, se observan posturas heterogéneas respecto a las funciones de la evaluación del aprendizaje en contextos de formación de agentes educativos. No se identifica una tendencia generalizada por parte del profesorado: mientras algunos abogan por las ventajas y beneficios que conlleva la función formativa, otros destacan un uso eminentemente sumativo de la información recolectada a través de los instrumentos de evaluación del aprendizaje. En coincidencia con lo anterior, se destacan las aportaciones realizadas por López, Benedito y León (2016), quienes a partir del análisis de las perspectivas de un grupo de expertos en pedagogía en el contexto español aseveran que la incorporación del enfoque por competencias en el nivel educativo superior representa una gran oportunidad para valorar la función formativa en las prácticas evaluativas. Los autores destacan que el uso de instrumentos tales como los exámenes teóricos no son útiles para la evaluación de competencias, remarcando otras opciones: portafolios, autoevaluación, coevaluación, estudio de caso, resolución de problemas, escalas y rúbricas. A su vez, frente a esta propuesta que destaca la función formativa de la evaluación del aprendizaje, Arribas Estebaranz (2017) sugiere comprender ambas funciones no como excluyentes sino que complementarias: se evalúa para mejorar y retroalimentar procesualmente el objeto evaluado, pero también para medir y tomar decisiones finales, dando forma al proceso evaluativo como tal.

Dentro de las limitaciones de la investigación, se debe destacar que se priorizó una aproximación exclusivamente cualitativa al objeto de estudio, lo cual permitió un acercamiento a la comprensión de las subjetividades de los participantes en torno a la evaluación del aprendizaje, pero impidió trazar conclusiones cuantitativas en torno a las percepciones docentes. Lo anterior dificulta el trazamiento de conclusiones tajantes en torno a la temática investigada, quedando vacíos respecto a si las categorías identificadas corresponden a las percepciones de la mayoría o minoría de los entrevistados. Frente a esto, se arguye que el análisis de la información recolectada se orientó a explorar de manera comprensiva los significados del profesorado, sin intentar generalizar las percepciones ni 
cuantificarlas. Para enfrentar estas limitaciones, se recomienda incluir métodos de investigación cuantitativos en los próximos estudios.

A nivel general, los hallazgos aportados por esta investigación permiten observar que en los procesos de enseñanza-aprendizaje apuntados a la formación de agentes educativos en ambos escenarios (UABC, México; UCM, España) se implementan procesos de evaluación del aprendizaje ligados a la funcionalidad mixta de la que se habla en los párrafos anteriores. Frente a esto, resulta insoslayable destacar la importancia que el profesorado le asigna a la función sumativa de la evaluación del aprendizaje, principalmente ligada a la toma de decisiones con base en los resultados de exámenes finales y a la certificación social. De esta manera, más allá de la trascendencia que se le otorga a la función formativa de la evaluación del aprendizaje a nivel teórico o discursivo, la práctica educativa habla de la persistencia de perspectivas sumativas difíciles de desarraigar (Moreno Olivos, 2007; INEE, 2011; García Sanz, 2014; Zitzelsberguer et al., 2017). De esta manera, se sugiere continuar con el análisis de las estrategias de evaluación del aprendizaje en los procesos de formación de agentes educativos, abarcando otros contextos de estudio, otras perspectivas y percepciones (estudiantes) y otros métodos de acercamiento a la realidad educativa estudiada (métodos cuantitativos).

\section{Referencias}

Álvarez Méndez, Juan Manuel. (2001). Evaluar para conocer, examiner para excluir. España: Morata.

Arribas Estebaranz, José María. (2017). La evaluación de los aprendizajes. Problemas y soluciones. Profesorado. Revista de Currículum y Formación del Profesorado, 21(4), 381-404. Recuperado de https://www.redalyc.org/pdf/567/56754639020.pdf

Carbajosa, Diana. (2011). Debates desde paradigmas en la evaluación educativa. Revista Perfiles Educativos, 33(128), 183-192.

Chiva, Inmaculada, Perales, María y Pérez Carbonell, Amparo. (2009). Historia de la Evaluación Educativa. En: Jornet, Jesús y Leyva, Yolanda. Conceptos, metodología y profesionalización en la evaluación educativa: México: INITE.

García Sanz, Mari Paz. (2014). La evaluación de competencias en educación superior mediante rúbricas: un caso práctico. Revista Electrónica Interuniversitaria de Formación del Profesorado REIFP, 1入1), 87-106. 
Hernández Sampieri, Roberto, Fernández Collado, Carlos y Baptista Lucio, Pilar. (2014). Metodología de la investigación. México: McGraw-Hill:

Instituto Nacional para la Evaluación de la Educación. (2011). Evaluación de los aprendizajes en el aula. Opiniones y prácticas de docentes de primaria en México. México: Autor.

Kamboj, Pooja y Kumar, Sushil. (2015). Effectiveness of Selected Teaching Strategiesin Relation to the Learning Styles of Secondary School Students in India. Interchange, 46, 289-312.

López, Carmen, Benedito, Vicente. y León, María. (2016). El enfoque de competencias en la formación universitaria y su impacto en la evaluación. La perspectiva de un grupo de profesionales expertos en pedagogía. Revista Formación Universitaria, 9(4), 11-22Recuperado de https://scielo.conicyt.cl/pdf/formuniv/v9n4/art03.pdf

Martínez, Elizabeth. (2004). Estrategias de enseñanza basadas en un enfoque constructivista. Revista Ciencias de la Educación, 2(24), 69-90.

Martínez Rizo, Felipe. (2012). Investigación empírica sobre el impacto de la evaluación formativa. Revisión de literatura. Revista Electrónica de Investigación Educativa, 14(1), 1-15. Recuperado de https://redie.uabc.mx/redie/article/view/291

Monereo, Carles, Castelló, Monserrat, Clariana, Mercè, Palma, Monserrat y Pérez, María. (2007). Estrategias de enseñanza y aprendizaje. Formación del profesorado y aplicación en la escuela. México: Graó.

Moreno Olivos, Tiburcio. (2007). La evaluación del aprendizaje en educación superior: El caso de la carrera de Derecho. Reencuentro, (48), 61-67.

Padilla Carmona, María Teresa y Gil Flores, Javier. (2008). La evaluación orientada al aprendizaje en Educación Superior: condiciones y estrategias para su aplicación en la docencia universitaria. Revista Española de Pedagogía, (241), 467-486.

Piñuel Raigada, José Luis. (2002). Epistemología, metodología y técnicas del análisis de contenidos. Estudios de Sociolingüística, 3(1), 1-42. Recuperado de https://www.ucm.es/data/cont/docs/268-2013-07-29-

Pinuel Raigada AnalisisContenido 2002 EstudiosSociolinguisticaUVigo.pdf

Ramos, Genoveva, Perales, María Jesús y Pérez Carbonell, Amparo. (2009). El concepto de evaluación educativa. En: Jornet, Jesús y Leyva, Yolanda, Conceptos, metodología y profesionalización en la evaluación educativa. México: INITE.

Robles, Bernardo. (2011). La entrevista en profundidad: una técnica útil dentro del campo antropofísico. Cuicuilco, 18(52), 39-49. Recuperado de http://www.redalyc.org/pdf/351/35124304004.pdf

Soriano Rodríguez, Ana María. (2012). Estrategias de enseñanza y evaluación en la universidad. Diá-logos, 10, 43-62. Recuperado de 
http://www.redicces.org.sv/jspui/bitstream/10972/2068/1/3.\%20Estrategias\%20de\%20e nsenanza\%20y\%20evaluacion\%20en\%20la\%20universidad.pdf

Vargas Jiménez, lleana. (2012). La entrevista en la investigación cualitativa: nuevas tendencias y retos. Revista Calidad en la Educación Superior, 3(1), 119-139. Recuperado de http://www.dialnet.unirioja.es/descarga/articulo/3945773.pdf

Zitzelsberguer, Hilde, Coffey, Sue, Graham, Leslie, Papaconstantinou, Efrosini y Anyman, Charles. (2017). Exploring Simulation Utilization and Simulation Evaluation Practices and Approaches in Undergraduate Nursing Education. Journal of Education and Practice, 8(3), 155-164. 
Revista indizada en

sciplo

redalyc latindex

DOAJ

DIRECTORY OF

JOURNALS

Distribuida en las bases de datos:

- Dialnet

$\mathrm{O}^{\circ}$ SHERPA/RøMEO

REDIB

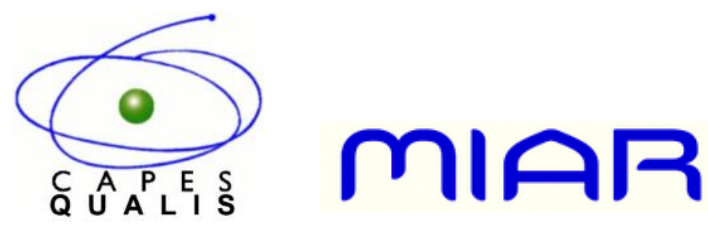

